\title{
MODELAGEM MATEMÁTICA E ANÁLISE DA HIDRATAÇÃO DE GRÃOS DE FEIJÃ̃ E LENTILHA SOB DIFERENTES TEMPERATURAS
}

Mathematical modeling and analysis of hydration of beans and lentil grains under different temperatures

Modelado matemático y análisis de la hidratación de granos de feijón y lentilha bajo diferentes temperaturas

\section{Bruna Araújo de Moura ${ }^{1}$, Lorena Brito Miranda ${ }^{1}$, Rômulo Alves Morais *2,3, Warley} Gramacho da Silva ${ }^{4}$, Glêndara Aparecida de Souza Martins 2,3

${ }^{1}$ Mestre em Ciência e Tecnologia de Alimentos, Universidade Federal do Tocantins, Palmas -TO, Brasil.

${ }^{2}$ Programa de Pós-Graduação em Ciência e Tecnologia de Alimentos, Universidade Federal do Tocantins, Palmas TO, Brasil.

${ }^{3}$ Laboratório de Cinética e Modelagem de Processos, Universidade Federal do Tocantins, Palmas - TO, Brasil.

${ }^{4}$ Departamento de Ciência da Computação, Universidade Federal do Tocantins, Palmas - TO, Brasil.

* Correspondência: Universidade Federal do Tocantins (UFT), Câmpus de Palmas, Avenida NS 15, Quadra 109

Norte/ Plano Diretor Norte Bloco II, sala 06, Palmas/TO, CEP: 77001-090.e-mail: romuloitallo2505@gmail.com

Artigo recebido em 29/04/2019 aprovado em 03/05/2019 publicado em 16/06/2019.

\section{RESUMO}

O feijão e a lentilha são leguminosas de papel importante na alimentação humana e ambas possuem nutrientes essenciais ao organismo. Desta forma faz-se necessário um estudo do comportamento destes grãos quando submetidos ao processo de hidratação, pois a maior parte das leguminosas é consumida na forma cozida. O objetivo deste trabalho foi avaliar a hidratação de duas cultivares de feijão e uma de lentilha, além de aplicar o modelo de Peleg nos dados obtidos. Para validar o modelo, foi realizada análise de umidade dos grãos de feijão branco, caupi e lentilha ao longo do tempo, por meio da imersão das cultivares em água destilada nas temperaturas de $25,35,45,55$ e $65^{\circ} \mathrm{C}$. O modelo de Peleg representou de forma mais satisfatória o processo de hidratação em todas as temperaturas estudadas para os grãos de feijão caupi e lentilha utilizando ajustes individuais dos seus parâmetros C1 e C2.

Palavras-chave: Leguminosas, absorção, modelo de Peleg.

\section{ABSTRACT}

Beans and lentils are legumes of important role in human nutrition and both have essential nutrients for the body. Therefore, it is necessary to study the behavior of these grains when submitted to the hydration process, since most of the legumes are consumed in the cooked form. The objective of this work was to evaluate the hydration of two bean and lentil cultivars, in addition to applying the Peleg model in the obtained data. To validate the model, a moisture analysis of the beans, cowpea and lentils was carried out over time, by immersing the cultivars in distilled water at temperatures of $25,35,45,55$ and $65^{\circ} \mathrm{C}$. The Peleg model represented in a more satisfactory way the hydration process in all temperatures studied for cowpea and lentil beans using individual adjustments of its parameters $C 1$ and $C 2$.

Keywords: Legumes, absorption, Peleg model.

\section{RESUMEN}

El frijol y la lenteja son leguminosas de papel importante en la alimentación humana y ambas poseen nutrientes esenciales al organismo. De esta forma se hace necesario un estudio del comportamiento de estos granos cuando 
sometidos al proceso de hidratación, pues la mayor parte de las leguminosas se consumen en la forma cocida. El objetivo de este trabajo fue evaluar la hidratación de dos cultivares de frijol y una de lenteja, además de aplicar el modelo de Peleg en los datos obtenidos. Para validar el modelo, se realizó un análisis de humedad de los granos de frijol blanco, caupí y lenteja a lo largo del tiempo, por medio de la inmersión de las cultivares en agua destilada a las temperaturas de 25, 35, 45, 55 y $65^{\circ} \mathrm{C}$. El modelo de Peleg representó de forma más satisfactoria el proceso de hidratación en todas las temperaturas estudiadas para los granos de frijol caupi y lenteja utilizando ajustes individuales de sus parámetros C1 y C2.

Palabras clave: Legumbres, absorción, modelo de Peleg.

\section{INTRODUÇÃO}

As leguminosas são grãos contidos em vagem, assim como feijão, lentilha e ervilha, ricas em vários nutrientes, como fibra, ferro, e com baixo teor de lipídeos (exceto amendoim e soja). A lentilha é altamente consumida pelas culturas orientais, na forma cozida ou como base para outros alimentos (ZIA-ULHAQ et al., 2011). Enquanto o feijão é muito apreciado pelos brasileiros, sendo uma das fontes básicas de proteína vegetal (BASSINELLO, 2008).

$\mathrm{O}$ enlatamento de leguminosas garante o consumo na entressafra e proporciona economia de tempo e energia, visto que são necessárias até 3 horas a $100^{\circ} \mathrm{C}$ para o cozimento de leguminosas, tais como a soja e o feijão (SIEGEl e FAWCETT, 1976; QUAST e SILVA, 1977).

Uma das formas de auxiliar nesse processo de industrialização é o estudo do comportamento destes grãos durante a hidratação, por meio de modelos matemáticos, sejam eles empíricos ou fenomenológicos. Um exemplo de modelo empírico bastante utilizado e recomendado para descrição da hidratação de leguminosas é o modelo de Peleg (GOWEN et al., 2007). A modelagem matemática permite predizer e simular o comportamento de determinado parâmetro e processos (LISBÔA, 2015). Adorno (2013) afirma que a "modelagem matemática aplicada à transferência de massa em alimentos visa à melhoria no processamento, redução de perdas de massa e energia e um aumento da qualidade do produto final."
Portanto, este trabalho tem por objetivo avaliar o processo de hidratação de duas espécies de feijão (Phaseolus vulgaris L.; Vigna unguiculata (L.) Walp) utilizando uma variedade de cada e uma espécie de lentilha (Lens Culinaris), além de aplicar o modelo de Peleg nos dados experimentais gerados.

\section{MATERIAIS E MÉTODOS}

Os grãos de feijão da espécie Phaseolus vulgaris L. e Vigna unguiculata L. Walp das variedades de feijão Branco, feijão Caupi, respectivamente, e sementes de lentilha da espécie Lens Culinaris foram adquiridos em mercado local.

Primeiramente realizou-se a seleção dos grãos, retirando os defeituosos e quaisquer impurezas contidas. Em seguida, foi realizada a análise de umidade dos grãos pelo método de estufa a $105 \pm 1^{\circ} \mathrm{C}$, por 24 horas em triplicata até massa constante (AOAC, 1995). O teor de umidade inicial encontrado para Feijão branco, Feijão Caupi e Lentilha foi de 0,08; 0,10; 0,09 (decimal b.s.) respectivamente.

Para avaliar o teor de água absorvida e a velocidade de absorção durante a hidratação dos grãos foram pesadas 50 unidades do grão e imersos em água destilada, numa relação de quatro volumes de água para um de produto, e levados a banho-maria, modelo TE-0541, a temperaturas controladas de 25, 35, 45, 55 e $65^{\circ} \mathrm{C}$ durante 14 horas ou até a completa hidratação do grão, realizadas em triplicata para cada temperatura. Em intervalos predeterminados, os grãos foram retirados dos beckeres e colocados em papel de filtro para eliminar os excessos de água, ficando de 
repouso por volta de $2 \mathrm{~min}$, em seguida pesados. Na sequência, os grãos retornavam à imersão.

Para avaliar o efeito da Temperatura e do Tempo de hidratação sobre o Teor de água absorvida e Velocidade de absorção dos grãos foi seguido um planejamento fatorial triplo. Os fatores avaliados foram: Tipo de grão $\mathrm{X}$ Temperatura $\mathrm{X}$ Tempo de hidratação.

Para modelagem do processo de hidratação dos grãos de feijão e lentilha, foi utilizado o modelo de Peleg (1988).

$$
U_{t}=U_{0}+\frac{t}{\left(C_{1}+C_{2} t\right)}
$$

Onde:

$\mathrm{U}_{\mathrm{t}}$ é o teor de água no tempo t, (decimal b.s.);

U0 é o teor de umidade inicial, (decimal b.s.);

t é o tempo de embebição, (h);

$C_{l}$ é a taxa constante de Peleg, (h decimal b.s. ${ }^{-1}$ );

$C_{2}$ é a capacidade constante de Peleg, (decimal b.s. ${ }^{-1}$ ).

Os dados das alterações nos Teores de água absorvida e Velocidade de absorção ocorridas nos grãos durante a hidratação foram analisados com o auxílio do aplicativo computacional SISVAR, por meio da análise de variância, adotando-se significância de 5\% de probabilidade para o teste $\mathrm{F}$, para testar as hipóteses dos efeitos principais e das interações. As alterações ocorridas devido ao efeito significativo do tempo e da temperatura foram explicadas pela análise de regressão, sendo que o melhor modelo ajustado foi escolhido através do coeficiente de determinação $\left(\mathrm{r}^{2}\right)$.

Para verificar o grau de ajuste do modelo de Peleg e da variação das constantes em função da temperatura de embebição, realizou-se uma análise de regressão pelo método Gauss Newton, utilizando-se o programa computacional Statistica 7.0®.

O grau de ajuste de cada modelo considerou a significância do coeficiente de regressão pelo teste $t$, adotando o nível de $1 \%$ de probabilidade, a magnitude do coeficiente de determinação $\left(\mathrm{R}^{2}\right)$, a magnitude do erro médio relativo $(\mathrm{P})$ e do erro padrão da estimativa (SE) que foram calculados pelas seguintes expressões:

$$
P=\frac{100}{n} \sum \frac{|Y-\hat{Y}|}{Y}
$$

$$
S E=\sqrt{\frac{\sum(Y-\hat{Y})^{2}}{G L R}}
$$

Onde, Y é o valor observado experimentalmente; $\hat{Y}$ é o valor estimado pelo modelo; n é o número de observações experimentais; GLR é o grau de liberdade do modelo (número de parâmetros do modelo subtraído do número de observações).

\section{RESULTADOS E DISCUSSÃO}

Os teores de água experimentais e estimados pelo modelo de Peleg, para os grãos de feijão branco, feijão caupi e lentilha, ao longo do processo de embebição para as diferentes temperaturas, estão apresentados na Figura 1. 
Figura 1. Teores de água experimentais e estimados pelo modelo de Peleg durante a hidratação dos grãos de feijão branco (a), caupi (b) e lentilha (c) nas temperaturas de $25,35,45,55$ e $65^{\circ} \mathrm{C}$.

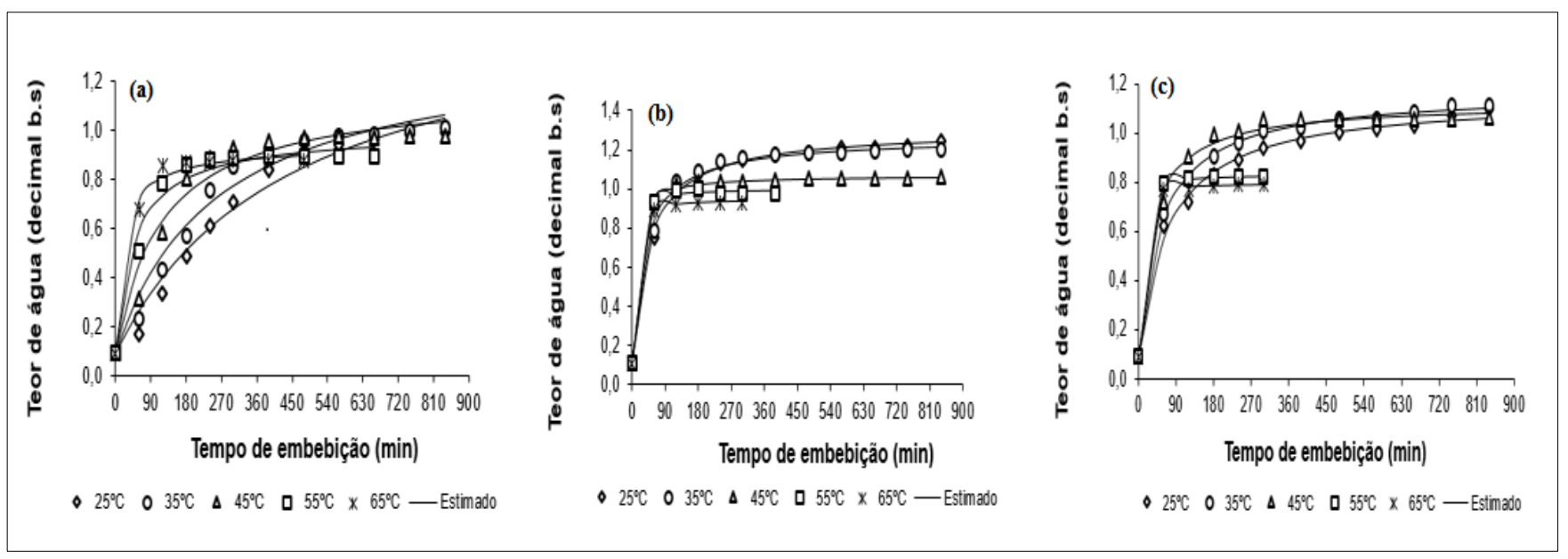

Tanto o feijão branco quanto o feijão caupi e a lentilha obtiveram um aumento significativo de absorção de água na fase inicial de hidratação. Segundo Resende e Corrêa (2007), este fato acontece, principalmente, devido à capilaridade natural existente nas camadas mais externas dos grãos, próximas ao hilo e ao processo de difusão.

O modelo de Peleg frente aos dados experimentais representou as principais tendências do processo de hidratação a partir do ajuste dos seus parâmetros para todos os grãos. No entanto, o melhor ajuste ocorreu para os grãos de feijão caupi e lentilha, que obtiveram correspondência entre os valores observados e estimados pelo modelo para todas as temperaturas de embebição $\left(25\right.$ a $\left.65^{\circ} \mathrm{C}\right)$.

Os valores dos coeficientes de determinação, erro médio relativo e erro padrão da estimativa para o modelo de Peleg, ajustado durante a hidratação do feijão Branco, feijão Caupi e lentilha nas temperaturas de $25,35,45,55$ e $65^{\circ} \mathrm{C}$, estão apresentados na Tabela 1.

Tabela 1. Valores do coeficiente de determinação $\left(R^{2}\right)$, erro médio relativo $(P)$, erro padrão da estimativa (SE), e coeficientes $(\mathrm{C} 1$ e $\mathrm{C} 2)$ para o modelo de Peleg ajustado durante a hidratação dos três tipos de grão nas diversas condições de temperatura.

\begin{tabular}{|c|c|c|c|c|c|c|}
\hline Grão & Temperatura ${ }^{\circ} \mathrm{C}$ & C1 & $\mathbf{C 2}$ & $\mathbf{R}^{2}(\%)$ & SE (decimal) & $P(\%)$ \\
\hline \multirow{5}{*}{ Feijão Branco } & 25 & $307,8138^{* * *}$ & $0,6795^{* *}$ & 97,73 & 0,0514 & 9,06 \\
\hline & 35 & $203,8416^{* *}$ & $0,7851^{* *}$ & 97,06 & 0,0579 & 8,44 \\
\hline & 45 & $111,6275^{* *}$ & $0,9292^{* * *}$ & 95,41 & 0,0669 & 8,11 \\
\hline & 55 & $53,9622^{* *}$ & $1,1051^{* *}$ & 97,35 & 0,0453 & 4,49 \\
\hline & 65 & $25,8321^{* *}$ & $1,1051^{* * *}$ & 98,96 & 0,0301 & 2,41 \\
\hline \multirow{5}{*}{ Feijão Caupi } & 25 & $38,4283^{* *}$ & $0,8389^{* * *}$ & 99,70 & 0,0183 & 1,28 \\
\hline & 35 & $30,8416^{* *}$ & $0,8654^{* *}$ & 99,48 & 0,0235 & 1,72 \\
\hline & 45 & $9,8008^{* *}$ & $1,0378^{* *}$ & 99,90 & 0,0082 & 0,48 \\
\hline & 55 & $4,2452^{* * *}$ & $1,1221^{* *}$ & 99,90 & 0,0134 & 0,88 \\
\hline & 65 & $4,8339^{* * *}$ & $1,1051^{* * *}$ & 99,98 & 0,0021 & 0,12 \\
\hline \multirow{5}{*}{ Lentilha } & 25 & $68,5885^{* *}$ & $0,9494^{* * *}$ & 99,22 & 0,0257 & 2,15 \\
\hline & 35 & $51,5610^{* *}$ & $0,9311^{* *}$ & 99,80 & 0,0128 & 1,04 \\
\hline & 45 & $32,3888^{* *}$ & $0,9695^{* * *}$ & 99,42 & 0,0222 & 1,74 \\
\hline & 55 & $4,2066^{* *}$ & $1,3537^{* *}$ & 99,98 & 0,0025 & 0,17 \\
\hline & 65 & $3,4994^{* *}$ & $1,4206^{* *}$ & 99,98 & 0,0035 & 0,24 \\
\hline
\end{tabular}

**Significativo ao nível de $1 \%$ de probabilidade pelo teste $\mathrm{t}$. 
Observa-se que os valores de coeficientes de determinação foram superiores a 95,41\% numa comparação de 0 a 100\%, o que revela a obtenção de valores elevados, considerando o erro médio relativo e o erro padrão de estimativa verifica-se um valor reduzido, abaixo de 10\% (Tabela 1).

Esse resultado obtido para as cinco temperaturas estudas indica que o modelo de Peleg apresentou significância dos seus parâmetros de regressão ao nível de $1 \%$ de probabilidade pelo teste $\mathrm{t}$. Caracterizando um ajuste adequado aos dados experimentais (CUNNINGHAM et al., 2007).
Para Turhan et al., (2002) o coeficiente $\mathrm{C} 1$ do modelo de Peleg está relacionado com a taxa de transferência de massa, e quanto menor for o seu valor, maior será a taxa inicial de absorção de água. Neste trabalho o coeficiente $\mathrm{C} 1$ decresceu à medida que aumentava a temperatura (Tabela 1), apresentando uma relação quadrática, em função da temperatura de hidratação.

Os resultados da análise de variância para as variáveis respostas em cinco temperaturas e seis tempos de hidratação estão apresentados na Tabela 2 para os grãos estudados.

\begin{tabular}{cccc}
\hline Causas de variação & \multicolumn{2}{c}{ Quadrado Médio das Variáveis } \\
\cline { 2 - 4 } Grão & $\mathrm{GL}$ & Teor de água absorvida & $\begin{array}{c}\text { Velocidade de } \\
\text { absorção } \\
\text { Temperatura }\end{array}$ \\
Tempo & 2 & $3393,7^{*}$ & $0,1325^{*}$ \\
Grão*Temperatura & 4 & $50,743^{*}$ & $0,0059^{*}$ \\
Grão*Tempo & 5 & $856,72^{*}$ & $0,0625^{*}$ \\
Temperatura*Tempo & 8 & $71,091^{*}$ & $0,0051^{*}$ \\
Grão*Temperatura*Tempo & 10 & $216,14^{*}$ & $0,0078^{*}$ \\
Erro & 20 & $8,4655^{*}$ & $0,0015^{*}$ \\
Total corrigido & 40 & $6,3927^{*}$ & $0,010^{*}$ \\
CV (\%) & 180 & & 9,95 \\
Média geral & 269 & & 0,0547 \\
\hline
\end{tabular}

Tabela 2. Resumo da análise de variância das variáveis respostas do processo de hidratação dos grãos.

* Significativo ao nível de 5\% pelo teste F.

Para todas as causas de variação os resultados apresentaram valores significativos $(\mathrm{p}<0,05)$ nas duas variáveis analisadas, o que mostra a existência de variabilidade entre as espécies testadas. Tanto o teor de água absorvida quanto a velocidade de absorção, demonstraram efeito significativo $(p<0,05)$ para todos os fatores fundamentais e nas interações.

\section{CONCLUSÃO}

Os três tipos de grãos estudados apresentaram características favoráveis para a produção de enlatados, levando em consideração o poder de absorção e devido o modelo de Peleg ter representado as principais tendências do processo, mas serão necessários mais estudos para determinar essa produção.

Todos os autores declararam não haver qualquer potencial conflito de interesses referente a este artigo.

\section{REFERÊNCIAS}

ADORNO, W. T., MARTINS, G. A. S., SILVA, W. S. Modelagem matemática aplicada a transferência de massa em alimentos. Enciclopédia Biosfera, Centro Científico Conhecer - Goiânia, v.9, N.16; 14 p., 2013. 

CHEMISTS (AOAC). Official methods of analysis of AOAC international. (16 ed.). Washington, 1995.

BASSINELLO, P. Z. Qualidade na escolha de variedades de feijão para o mercado consumidor. Atualidades em micotoxinas e armazenagem qualitativas de grãos II (1. ed.). Florianópolis: Imprensa Universitária, 2008.

CUNNINGHAM, S. E., MCMINN, W. A. M., MAGEE, T. R. A., RICHARDSON, P. S. Modelling water absorption of pasta during soaking. Journal of Food Engineering, v. 82, ed. 4, p. 600-607, 2007.

GOWEN, A., ABU-GHANNAM, N., FRIAS, J., OLIVEIRA, J. Modelling the water absorption process in chickpeas (Cicer arietinum L.)The effect of blanching pre-treatment on water intake and texture kinetics. Journal of Food Engineering, v. 78, n. 3, p. 810-819, 2007.

LISBÔA, J. F., SILVA, J. N., CAVALCANTI, M. T., SILVA, E. M. C., GONÇALVES, M. C. Análise da hidratação de grãos de alpiste. $R$. Bras. Eng. Agríc. Ambiental, v.19, n.3, p.218-223, 2015.

PELEG, M. An empirical model for the description of moisture sorption curves. Journal of Food Engineering, London, v. 52, p. 1216-1219, 1988.

Quast, D.C., Silva S. D. Temperature dependence of the cooking rate of dry legumes. Journal of Food Science, Chicago, v. 42, n. 2, p. 370-374, 1977.

RESENDE, O., CORRÊA, P. C. Modelagem matemática do processo de hidratação de sementes de feijão. Acta Scientiarum Agronomy, v. 29, n. 3, p. 373-378, 2007.

SIEGEL, A., FAWCETT, B. Food legume processing and utilization. Ottawa: IDRC. 88p. 1976.

TURHAN, M., SAYAR, S.; GUNASEKARAN, S. Application of Peleg model to study water absorption in chickpea during soaking. Journal of Food Engineering, v. 53, p. 153-159, 2002.

ZIA-UL-HAQ, M., AHMAD ,S., SHAD, M.A., IQBAL, S., QAYUM, M., AHMAD, A., LUTHRIA, D.L., AMAROWICZ, R. Compositional studies of some of lentil cultivars commonly consumed in Pakistan. Pakistan. J. Bot., 43(3): 1563-1567, 2011. 